\title{
Mechanisms regulating follicle wave patterns in the bovine estrous cycle investigated with a mathematical model
}

\author{
H. M. T. Boer,${ }^{*} \dagger^{1}$ S. Röblitz, $\ddagger$ C. Stötzel, $\neq$ R. F. Veerkamp, ${ }^{*}$ B. Kemp, $†$ and H. Woelders* \\ *Animal Breeding and Genomics Centre, Wageningen UR Livestock Research, 8200 AB Lelystad, the Netherlands \\ †Adaptation Physiology Group, Department of Animal Sciences, Wageningen University, 6700 AH Wageningen, the Netherlands \\ ‡Zuse Institute Berlin (ZIB), Department of Numerical Analysis and Modeling, Computational Systems Biology Group, Takustraße 7 , \\ 14195 Berlin, Germany
}

\section{ABSTRACT}

A normal bovine estrous cycle contains 2 or 3 waves of follicle development, and ovulation takes place in the last wave. However, the biological mechanisms that determine whether a cycle has 2 or 3 waves have not been elucidated. In a previous paper, we described a mathematical model of the bovine estrous cycle that generates cyclical fluctuations of hormones, follicles, and corpora lutea in estrous cycles of approximately 21 $\mathrm{d}$ for cows with a normal estrous cycle. The parameters in the model represent kinetic properties of the system with regard to synthesis, release, and clearance of hormones and growth and regression of follicles and corpora lutea. The initial model parameterization resulted in estrous cycles with 3 waves of follicular growth. Here, we use this model to explore which physiological mechanisms could affect the number of follicular waves. We hypothesized that some of the parameters related to follicle growth rate or to the time point of corpus luteum regression are likely candidates to affect the number of waves per cycle. We performed simulations with the model in which we varied the values of these parameters. We showed that variation of (combinations of) model parameters regulating follicle growth rate or time point of corpus luteum regression can change the model output from 3 to 2 waves of follicular growth in a cycle. In addition, alternating 2- and 3-wave cycles occurred. Some of the parameter changes seem to represent plausible biological mechanisms that could explain these follicular wave patterns. In conclusion, our simulations indicated likely parameters involved in the mechanisms that regulate the follicular wave pattern, and could thereby help to find causes of declined fertility in dairy cows.

Key words: mathematical model, estrous cycle, follicular wave pattern

Received March 28, 2011.

Accepted August 27, 2011.

${ }^{1}$ Corresponding author: Marike.Boer@wur.nl

\section{INTRODUCTION}

Two different patterns of follicle development are identified in mammals. In humans (and rats and pigs), the development of follicles to ovulatory size occurs only during the follicular phase, whereas in cattle (and sheep and horses), development of follicles to ovulatory or near-ovulatory size occurs throughout the cycle (Fortune, 1994). A normal bovine estrous cycle consists of 2 or 3 waves in which a cohort of follicles starts to grow. Many studies report mostly 2-wave cycles (Ahmad et al., 1997; Bleach et al., 2004; Wolfenson et al., 2004; Burns et al., 2005; Jaiswal et al., 2009). Cycles with 1 or 4 waves occur incidentally (Bleach et al., 2004; Wolfenson et al., 2004). In both 2- and 3-wave cycles, serum levels of FSH start to increase directly after ovulation (d 0 ), inducing the emergence of the first follicular wave. Typically, emergence of the second wave occurs on d 9 to 10 in 2-wave cycles and on d 8 to 9 in 3 -wave cycles. In 3 -wave cycles, a third wave emerges on d 15 to 16 . The first 1 or 2 waves produce a dominant follicle that does not ovulate but undergoes regression under influence of progesterone (P4). The functional dominant follicle present at the onset of luteolysis becomes the ovulatory follicle. Regression of the corpus luteum (CL) starts earlier in 2-wave cycles (d 16) than in 3-wave cycles (d 19), resulting in cycle lengths of 19 to $20 \mathrm{~d}$ and 22 to $23 \mathrm{~d}$, respectively (reviewed by Adams et al., 2008).

The reason for cycles being of the 2- or 3-wave type is unclear. No difference was found between cows and heifers with regard to the proportion of 2- and 3-wave cycles (Wolfenson et al., 2004). The number of waves in a cycle does not appear to be affected by breed or age (reviewed by Adams et al., 2008). An increase in 3 -wave patterns has been associated with poor nutrition and heat stress (reviewed by Adams et al., 2008). A higher milk yield was reported in cows with 2 -wave cycles (Bleach et al., 2004). Several experiments have been performed to search for endocrine mechanisms underlying 2-wave versus 3 -wave cycles. One possible explanation of a difference in number of waves is a dif- 
ference in CL life span. The onset of CL regression occurs $2.5 \mathrm{~d}$ earlier in 2 -wave than in 3 -wave cycles (Ahmad et al., 1997; Jaiswal et al., 2009). Another possible explanation is that slowly growing dominant follicles delay the start of the next wave. Ovulatory follicles in 2-wave cycles have a lower growth rate than ovulatory follicles in 3-wave cycles (Bleach et al., 2004). Cauterization of the dominant follicle of the first wave [i.e., reduced estradiol (E2) and inhibin (Inh) production] at $\mathrm{d} 3$ or 5 of the cycle resulted in an FSH surge the day after cauterization (Adams et al., 1992) and an earlier emergence of the next wave (Ko et al., 1991). Cows with 3-wave cycles had lower serum FSH and Inh concentrations in nonovulatory waves compared with cows with 2-wave cycles (Parker et al., 2003), and it was therefore suggested that the number of waves during the cycle is affected by serum FSH and Inh concentrations. The latter is confirmed by the finding that immunization against Inh-A increased the number of follicular waves during a cycle (Medan et al., 2006).

Mathematical modeling of the bovine estrous cycle could help in understanding the dynamics of this complex biological system. Recently, we developed a mathematical model of the bovine estrous cycle (Boer et al., 2011). The objective of this paper was to investigate which mechanisms could be likely candidates for regulation of the number of waves in the bovine estrous cycle, using this model. A better understanding of the endocrine mechanisms regulating follicle development could help to find causes of declined fertility in dairy cows.

\section{MATERIALS AND METHODS}

\section{Parameterization of Follicle Wave Patterns}

In cattle, the functional follicle that is dominant at the time of CL regression develops to become the ovulatory follicle. We assumed that 2 mechanisms may exist by which follicle wave pattern can be influenced. One is the rate of follicle growth and the other is the time point of CL regression. The first mechanism might be induced by changing the effect of FSH or P4 on follicle growth, or by changing FSH or P4 synthesis, because follicle growth is stimulated by FSH and inhibited by P4 (Ko et al., 1991; Parker et al., 2003; Medan et al., 2006). The second mechanism; that is, the time point of CL regression, is expected to have an effect on the follicular wave pattern because 2-wave cycles can occur when the CL starts to regress at an earlier time point; for example, because of an earlier increase of $\mathrm{PGF}_{2 \alpha}$ (Adams et al., 1992; Ahmad et al., 1997; Jaiswal et al., 2009). We selected 10 parameters in our model (Table 1), of which 7 relate to the first mechanism and 3 to the second mechanism, and we tested whether changing the value of these parameters affects the number of waves per cycle in the model simulations. The model was simulated for $120 \mathrm{~d}$ and can be simulated for as many days as required. For each of the 10 parameters, we performed simulations in which we changed the parameter with small steps for a range of values around the initial value. Results were evaluated by studying the figures of the model solution. We did not further increase or decrease the value when the solution continued to be irregular. The rationale for the expected effect of a parameter on either follicle growth rate or time point of CL regression is given in more detail in the Results section, together with the simulation results for that parameter.

A sensitivity analysis for the complete model has been performed with the techniques described in Deuflhard (2004) and Grah (2004). Sensitivities have been calculated with external numerical differentiation of the system at specified time points. These sensitivities were obtained as difference quotients depending on the solution components and parameter perturbations. The perturbations were taken as $0.1 \%$ of the absolute parameter values or at least $10 \mathrm{e}^{-5}$. The values were then weighted according to parameter ranges and measurement information to obtain the scaled sensitivity matrix. The column norms of the sensitivity matrix represent the relative sensitivities of the system with regard to each parameter. A higher column norm indicates a parameter whose change in value has a larger effect on the model solution compared with changes of other parameter values, which means that this parameter is a more sensitive parameter.

\section{Brief Description of the Model}

Recently, we developed a deterministic mathematical model that describes the dynamics of the bovine estrous cycle as a set of linked differential equations, including the processes of follicle and CL development and the working of key hormones that interact to control these processes (Boer et al., 2011). In the differential equations, Hill functions (Boer et al., 2011) were used for the modeling of inhibitory and stimulatory effects of hormones. A Hill function is a sigmoidal function between 0 and 1, which switches at a specified threshold from one level to the other with a specified steepness. Time delays were incorporated when appropriate, to capture the time needed for factors to influence each other. Parameter estimation was based on experimental measurements available in the literature. The simulations of this model are in line with empirical knowledge. With the parameterization of Boer et al. (2011), the model generates estrous cycles of $21 \mathrm{~d}$, with 3 peaks 
Table 1. Parameter description, initial parameter value, and hypothesized direction of change (increase or decrease) to alter a 3-wave in a 2-wave cycle for the 10 parameters that were tested for an effect on follicular wave pattern ${ }^{1}$

\begin{tabular}{|c|c|c|c|c|}
\hline $\begin{array}{l}\text { Parameter } \\
\text { no. }\end{array}$ & $\begin{array}{l}\text { Parameter } \\
\text { symbol }^{2}\end{array}$ & Parameter explanation & $\begin{array}{c}\text { Initial } \\
\text { value }\end{array}$ & Change \\
\hline 1 & $T_{F S H}^{F o l l}$ & Threshold of FSH above which the stimulating effect on Foll is increased & 1.44 & $\uparrow$ \\
\hline 3 & $m_{P 4}^{\text {Foll }}$ & Maximum inhibition rate on Foll of $\mathrm{P} 4$ & 2.17 & $\downarrow$ \\
\hline 4 & $T_{\text {Foll }}^{\text {FSH }}$ & $\begin{array}{l}\text { Threshold of Foll above which the stimulating effect of FSH on Foll decreases } \\
\text { [larger follicles become more sensitive to (i.e., less dependent on) FSH] }\end{array}$ & 0.30 & $\downarrow$ \\
\hline 5 & $c_{\text {Foll }}^{\text {Inh }}$ & Proportionality factor of Foll to Inh (the production of Inh is proportional to Foll) & 4.33 & $\uparrow$ \\
\hline 6 & $T_{I n h}^{F S H}$ & Threshold of Inh above which the inhibiting effect on FSH synthesis is increased & 0.06 & $\downarrow$ \\
\hline 7 & $m_{I n h}^{F S H}$ & Maximum FSH synthesis rate in absence of Inh & 1.46 & $\downarrow$ \\
\hline 8 & $c_{C L}^{P 4}$ & $\begin{array}{l}\text { Proportionality factor of CL to P4 (the production } \\
\text { of P4 is proportional to CL function) }\end{array}$ & 0.50 & $\downarrow$ \\
\hline
\end{tabular}

\footnotetext{
${ }^{1}$ Parameter values are on a relative scale to simplify parameter estimation.

${ }^{2} T$ denotes a threshold, $m$ denotes a maximum, and $c$ denotes a rate constant; Foll $=$ follicular function, $\mathrm{P} 4=$ progesterone, Inh $=$ inhibin, CL $=$ corpus luteum, OTR $=$ oxytocin receptor and enzymes required for $\mathrm{PGF}_{2 \alpha}$ production.
}

of FSH and 3 corresponding waves of follicle growth and Inh production. In the model, $\boldsymbol{C L}$ denotes the CL function; that is, the capacity of the CL to produce $\mathrm{P} 4$, rather than the physical size of the CL. Likewise, follicular function (Foll) represents the combined capacity of all follicles present at any time to produce E2 and Inh. Each follicular wave is induced by an increase in FSH. Progesterone, which is high during the first 2 waves, decreases as the CL regresses under influence of $\mathrm{PGF}_{2 \alpha}$ released from the uterus. The third wave of follicle growth then results in increasing levels of E2. This causes a surge of $\mathrm{GnRH}$ and hence LH, which triggers ovulation (Figures 1 and 2). More details about the incorporated physiological mechanisms and the mathematical description of the model can be found in Boer et al. (2011).

\section{Modification of the Model}

The model described in Boer et al. (2011) includes 2 fixed time delays for the effect of the increase in P4 levels on $\mathrm{PGF}_{2 \alpha}$ release, to mimic the situation that the increase of $\mathrm{P} 4$ early in the cycle starts a series of events or mechanisms that eventually leads to the increase of $\mathrm{PGF}_{2 \alpha}$, followed by a decline of $\mathrm{PGF}_{2 \alpha}$ a few days later. The time delays are thus a "black box" in which the intermediate events that regulate $\mathrm{PGF}_{2 \alpha}$ levels are not described. We have adapted this part of the model. In the model used for the current study, these delays are replaced by a mechanism in which the ability to synthesize $\mathrm{PGF}_{2 \alpha}$ develops over time under influence of P4. Exposure to P4 above a threshold level must last for a couple of days to induce $\mathrm{PGF}_{2 \alpha}$ release. Progesterone stimulates the synthesis of receptors (e.g., oxytocin receptor, OTR) and enzymes required for the production and release of $\mathrm{PGF}_{2 \alpha}$ (Silvia et al., 1991; dos Santos et al., 2009). In the model, OTR thus represents the overall mechanism in the endometrium involved in the production of $\mathrm{PGF}_{2 \alpha}$; OTR is stimulated by $\mathrm{P} 4$,

$$
\frac{\mathrm{d}}{\mathrm{d} t} \operatorname{OTR}(t)=c_{P 4}^{O T R} \cdot P 4(t)-c_{O T R} \cdot \operatorname{OTR}(t),
$$

where $c_{P 4}^{O T R}$ is the proportionality factor of $\mathrm{P} 4$ in the increase of OTR, $c_{\text {OTR }}$ is the decrease rate of OTR, and $(t)$ denotes time; OTR stimulates $\mathrm{PGF}_{2 \alpha}$,

$$
\frac{\mathrm{d}}{\mathrm{d} t} P G F 2 \alpha(t)=H^{+}(O T R)-c_{P G F 2 \alpha} \cdot P G F 2 \alpha(t),
$$

where $\mathrm{H}^{+}(\mathrm{OTR})$ is the Hill function of the stimulating $(+)$ effect of OTR, and $c_{P G F 2 \alpha}$ is the $\mathrm{PGF}_{2 \alpha}$ clearance rate constant. Levels of $\mathrm{PGF}_{2 \alpha}$ thus increase because P4 stimulates the production of OTR required for $\mathrm{PGF}_{2 \alpha}$ production (Figures 1 and 2). Figure 1 shows an overview of the mechanisms incorporated in the current model. The equations and parameter values are listed in the Appendix (Tables A1, A2, and A3). The model contains 13 differential equations and 54 parameters. 
A.

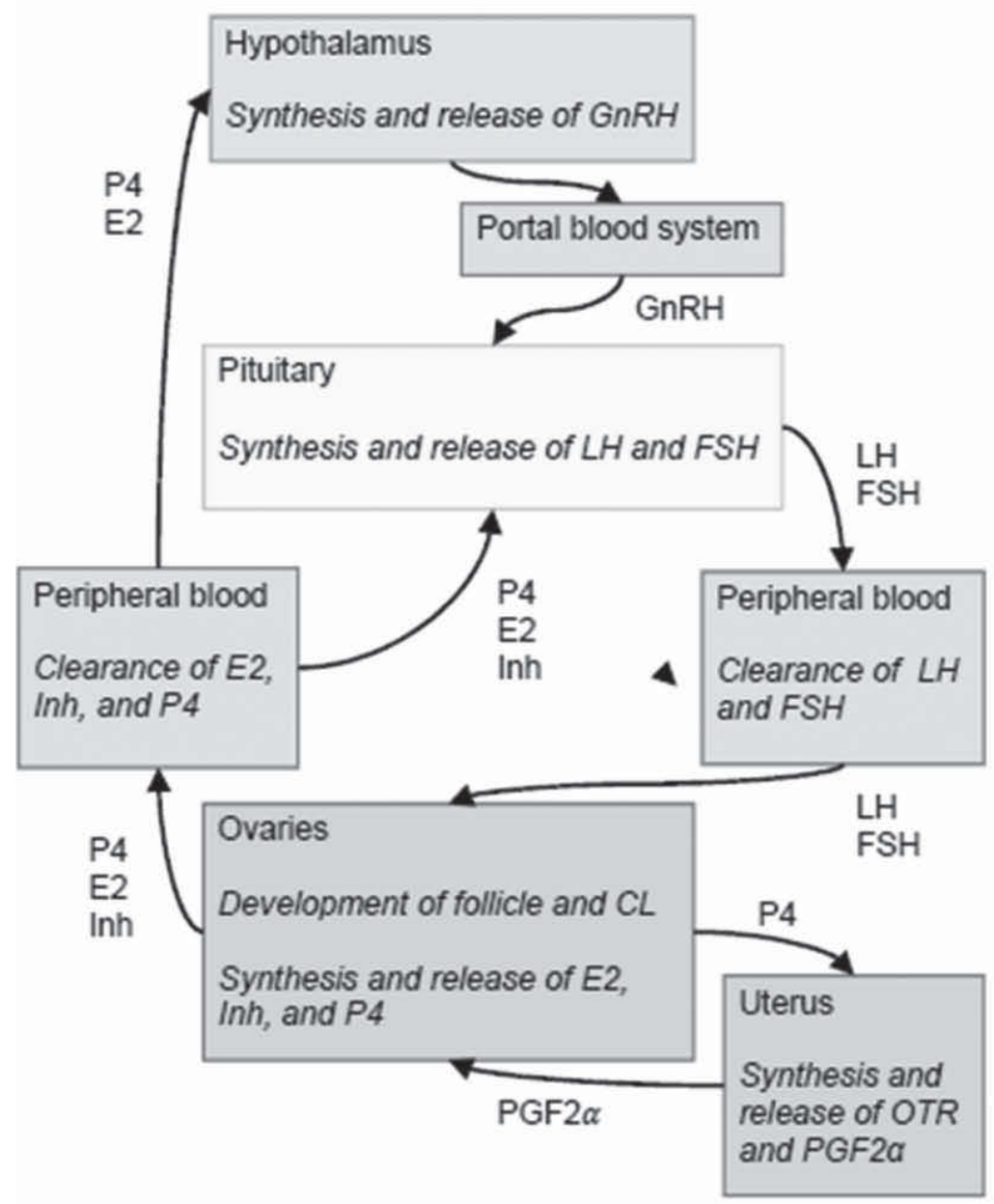

B.

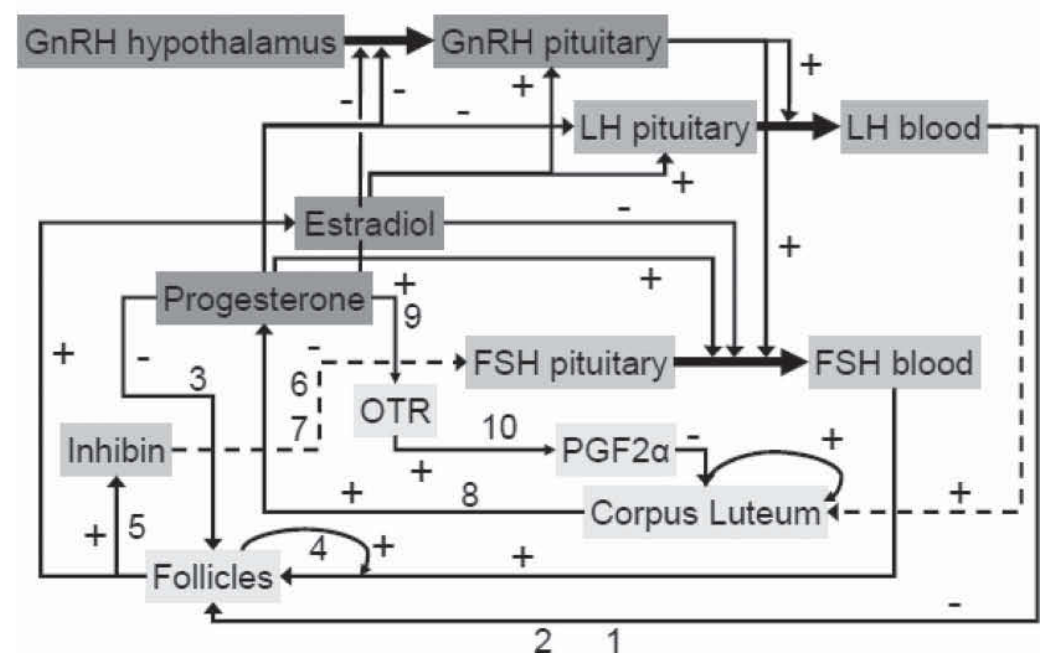

Figure 1. (A) Compartmental representation of the basis for the mathematical model of the bovine estrous cycle. Each process is represented by a set of equations. (B) Mechanisms of the model of the bovine estrous cycle, in which the boxes represent the 13 components for which a differential equation was derived. Stimulating and inhibiting effects are denoted by '+' and '-', respectively; dashed lines denote a time delay. The numbers (as used in Table 1) of the parameters that were changed in the simulations of this study are placed at the mechanism (arrow) that they affect. $\mathrm{P} 4=$ progesterone, $\mathrm{E} 2=$ estradiol, $\mathrm{Inh}=$ inhibin, $\mathrm{OTR}=$ oxytocin receptor and enzymes required for $\mathrm{PGF}_{2 \alpha}$ production 

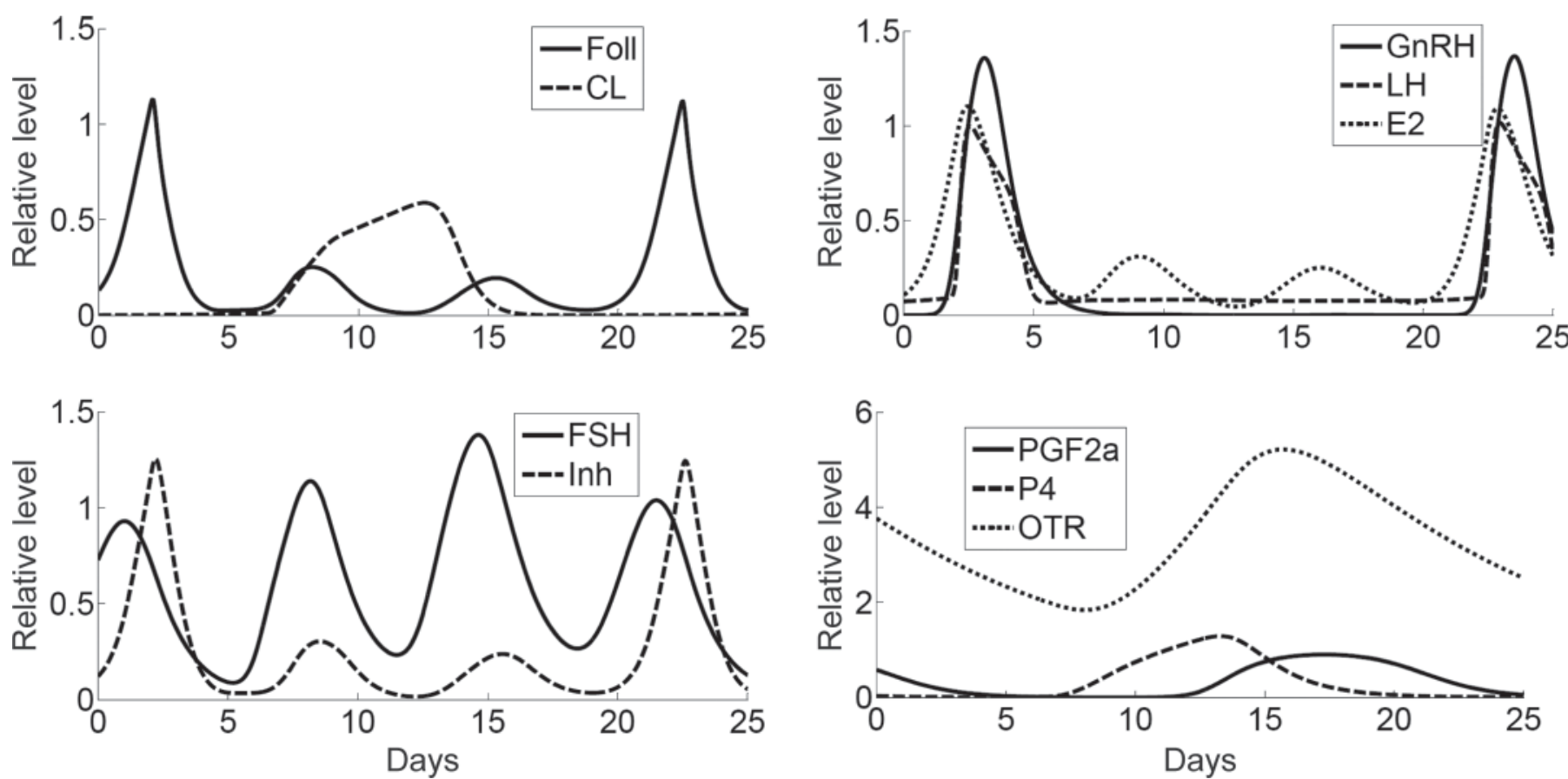

Figure 2. The initial model parameterization results in estrous cycles with 3 waves of follicular growth. The equations are expressed on a relative scale to simplify parameter estimation, and therefore the $\mathrm{y}$-axis of the figures is dimensionless. Foll $=$ follicular function, CL $=$ corpus luteum function, $\mathrm{E} 2=$ estradiol, $\mathrm{Inh}=$ inhibin, $\mathrm{P} 4=$ progesterone, OTR $=$ oxytocin receptor.

\section{RESULTS AND DISCUSSION}

Of the 10 parameters tested, 6 affected the number of waves per cycle (Table 2). Cycles with 2 waves had a shorter cycle length. In the nonovulatory wave of 2-wave cycles, FSH levels were higher, Foll (follicular capacity to produce E2 and Inh) was larger, and therefore E2 and Inh levels were also higher compared with nonovulatory waves of 3 -wave cycles. The 2 -wave cycles obtained by a change in follicle growth rate were due to a later emergence of the second wave, whereas the 2 -wave cycles obtained by a change in time point of CL regression were caused by a shorter CL life span.

\section{Effects of Parameters Related to Follicle Growth Rate}

Effect of $\boldsymbol{F S H}$. Follicle growth rate is stimulated by FSH, and the FSH-dependent growth rate in the model is influenced by several parameters: for example, maximum rate of FSH-dependent growth $\left(m_{F S H}^{F o l l}\right)$ and threshold of FSH to stimulate Foll $\left(T_{F S H}^{F o l l}\right)$. The parameters $m_{F S H}^{\text {Foll }}$ and $T_{F S H}^{\text {Foll }}$ belong to the Hill function of the effect of FSH on Foll, where the first represents the maximum FSH-dependent follicle growth rate, and the second defines the threshold of FSH at which the stimulatory effect on Foll increases. We tested whether a lower follicle growth rate could be simulated by a decrease in
$m_{F S H}^{F o l l}$. In the simulations, a decrease in $m_{F S H}^{F o l l}$ alone did not result in a 2 -wave cycle, but the wave pattern could be changed in combination with another parameter involved in follicle growth rate, maximum inhibition rate of $\mathrm{P} 4$ on Foll $\left(m_{P 4}^{\text {Foll }}\right)$. A decrease in $m_{F S H}^{\text {Foll }}$ from 0.70 to 0.40 resulted in 2-wave cycles when, at the same time, $m_{P 4}^{F o l l}$ was decreased, and the resulting Foll peak height in nonovulatory waves was higher (Figure 3). Apparently, despite the lower FSH-dependent growth rate, the first wave could then grow larger and persist for longer due to the lower inhibiting effect of P4. Therefore, the second wave occurred when the CL was already waning, allowing the second wave to become the ovulatory wave.

Remarkably, increasing $m_{F S H}^{F o l l}$ from 0.70 to 1.35 (without a change in $m_{P 4}^{F o l l}$ ) resulted in alternating 2-wave and 3-wave cycles (Figure 4). Increasing $m_{F S H}^{\text {Foll }}$ even further, to 1.80 , resulted in a series of 2 -wave cycles. However, LH peaks then became irregular, which appeared to be related to the increased E2 levels produced by the larger follicles. Decreasing the sensitivity of the pituitary for $\mathrm{E} 2$ resulted in normal LH peaks again. This was done by increasing the E2 threshold for GnRH sensitivity of the pituitary $\left(T_{E 2}^{G n R H, 2}\right)$, which is the threshold of E2 at which the pituitary becomes more sensitive to GnRH. When $T_{E 2}^{G n R H, 2}$ was increased from 0.88 to 1.62 , the $\mathrm{GnRH} / \mathrm{LH}$ surge was induced at the 
5992

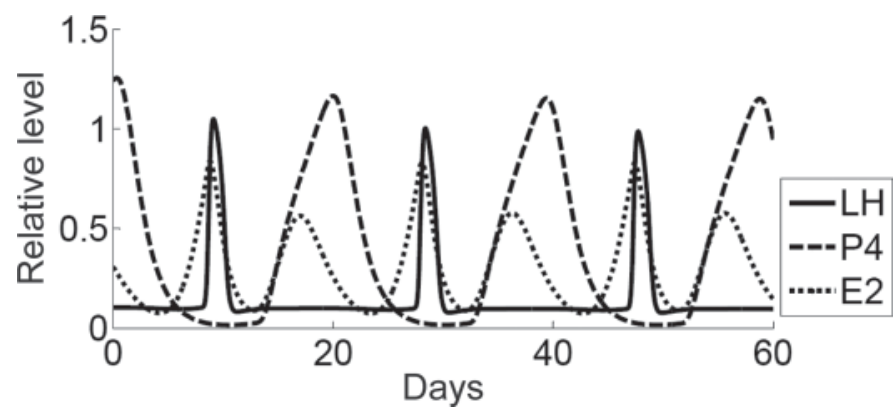

Figure 3. A decreased maximum rate of FSH-dependent growth $\left(m_{F S H}^{\text {Foll }}\right)$ combined with a decreased maximum inhibition rate of $\mathrm{P} 4$ on Foll $\left(m_{P 4}^{\text {Foll }}\right)$ resulted in cycles with 2 follicular waves. The equations are expressed on a relative scale to simplify parameter estimation, and therefore no dimension is given at the $\mathrm{y}$-axis of the figures. $\mathrm{P} 4=$ progesterone, $\mathrm{E} 2$ = estradiol.

appropriate time point; that is, together with E2 peak levels. Likely, an increase of $m_{F S H}^{\text {Foll }}$ (instead of the expected decrease) results in a 2 -wave cycle because it takes longer before the inhibiting effect of $\mathrm{P} 4$ becomes stronger than the increased stimulating effect of FSH, and therefore the first follicular wave starts to decline at a later time point. We tested whether a lower follicle growth rate could be simulated by an increase in $T_{F S H}^{F o l l}$. However, a change in $T_{F S H}^{F o l l}$ did not affect wave pattern.

In the model, larger follicles are less dependent on FSH; that is, follicles larger than the threshold $T_{\text {Foll }}^{F S H}$ (threshold of Foll to downscale FSH threshold) become

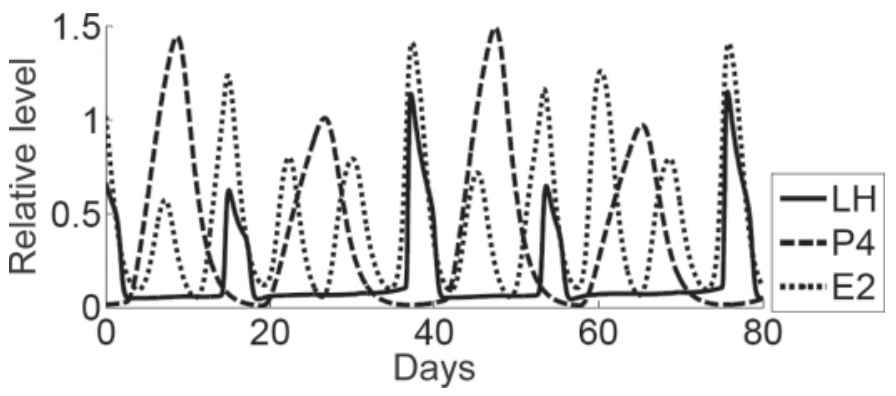

Figure 4. When the maximum rate of FSH-dependent growth $\left(m_{F S H}^{\text {Foll }}\right)$ was increased from 0.70 to 1.35 , the model simulated alternately 2 -wave and 3 -wave cycles. $\mathrm{P} 4=$ progesterone, $\mathrm{E} 2=$ estradiol.

more sensitive to (i.e., less dependent on) FSH. Therefore, we tested whether a decrease in $T_{F o l l}^{F S H}$ would lead to fewer waves per cycle. Simulation results showed that this parameter indeed affected the wave pattern. When $T_{\text {Foll }}^{F S H}$ was decreased from 0.30 to 0.15 , the model simulated alternating 2 -wave and 3 -wave cycles, with some variation in hormone levels between cycles. When this parameter was 0.12 , almost all cycles had 2 waves (Figure 5). With values below 0.12, E2 and LH patterns became irregular.

Effect of $\boldsymbol{P} 4$. We assumed that when follicle growth is less inhibited by $\mathrm{P} 4$, follicles in the luteal phase could grow larger and persist for longer, which could result in a higher occurrence of cycles with 2 waves. We have tested this hypothesis by decreasing the maximum inhi-

Table 2. Overview of whether a change in parameter value affects the number of waves per cycle and of the changes in parameter values that resulted in a different number of waves

\begin{tabular}{|c|c|c|c|c|c|}
\hline $\begin{array}{l}\text { Parameter } \\
\text { symbol }^{1}\end{array}$ & $\begin{array}{l}\text { Change in } \\
\text { wave number }\end{array}$ & $\begin{array}{l}\text { Initial } \\
\text { value }^{2}\end{array}$ & $\begin{array}{l}\text { Adapted } \\
\text { value }\end{array}$ & $\begin{array}{l}\text { Number } \\
\text { of waves }\end{array}$ & Remark \\
\hline$T_{F S H}^{F o l l}$ & No & 1.44 & & & \\
\hline \multirow[t]{2}{*}{$m_{F S H}^{\text {Foll }}$} & \multirow[t]{2}{*}{ Yes } & \multirow[t]{2}{*}{0.70} & 0.40 & 2 & When $m_{P 4}^{\text {Foll }}$ is 0.50 \\
\hline & & & 1.80 & 2 & When $T_{E 2}^{G n R H, 2}$ is increased from 0.88 to 1.62 \\
\hline \multirow[t]{2}{*}{$m_{P 4}^{\text {Foll }}$} & \multirow[t]{2}{*}{ Yes } & \multirow[t]{2}{*}{2.17} & 1.00 & 2 & \\
\hline & & & 1.45 & 2 and $3^{3}$ & \\
\hline$T_{\text {Foll }}^{F S H}$ & Yes & 0.30 & 0.12 & 2 & Almost all cycles 2 waves \\
\hline$c_{\text {Foll }}^{\text {Inh }}$ & No & 4.33 & & & \\
\hline$T_{I n h}^{F S H}$ & Yes & 0.06 & 0.30 & 2 & Irregular FSH and Inh levels \\
\hline$m_{\text {Inh }}^{F S H}$ & No & 1.46 & & & \\
\hline$c_{C L}^{P 4}$ & No & 0.50 & 1.45 & 3 & When $m_{P 4}^{\text {Foll }}$ is 1.00 \\
\hline$c_{P 4}^{O T R}$ & Yes & 0.87 & 2.00 & 2 & \\
\hline \multirow[t]{2}{*}{$T_{O T R}^{P G F 2 \alpha}$} & \multirow[t]{2}{*}{ Yes } & \multirow[t]{2}{*}{3.97} & 2.00 & 2 & \\
\hline & & & 2.50 & 2 and $3^{3}$ & \\
\hline
\end{tabular}

${ }^{1}$ See Table 1 for definition of parameters.

${ }^{2}$ The initial parameter value results in 3 -wave cycles.

${ }^{3}$ Two- and 3 -wave cycles alternating. 
bition rate of $\mathrm{P} 4$ on Foll $\left(m_{P 4}^{\text {Foll }}\right)$, which is the parameter that represents the maximum inhibiting effect of $\mathrm{P} 4$ on follicular function. The model generated cycles with 2 waves when $m_{P 4}^{\text {Foll }}$ was between 0.60 and 1.20. When $m_{P 4}^{\text {Foll }}$ was between 1.20 and 1.80 , the cycle contained sometimes 2 and sometimes 3 waves, or somewhat irregular patterns. When $m_{P 4}^{\text {Foll }}$ was 1.80 or higher, the model generated cycles with 3 waves, and the peak levels of Foll in nonovulatory waves became lower with further increased $m_{P 4}^{\text {Foll }}$ (because the inhibiting effect of P4 became stronger) until the cycle contained basically 1 ovulatory wave, without waves during the luteal phase.

FSH Synthesis. Another possible mechanism that may affect follicle growth rate is FSH synthesis. We assumed that a stronger inhibiting effect of Inh on FSH synthesis could result in lower FSH serum levels and therefore slower follicle growth, resulting in a cycle with 2 instead of 3 waves. We tested whether a decrease in maximum FSH synthesis rate in the pituitary $\left(m_{\text {Inh }}^{F S H}\right)$ could reduce the FSH-dependent follicle growth rate and result in 2-wave cycles. However, a decrease in this parameter did not result in a regular 2 -wave cycle, but in low peak levels of Foll without LH surge. This was due to E2 levels that were too low to induce the $\mathrm{GnRH} /$ LH surge. The E2-dependent GnRH/LH surge could not be repaired by a decrease in $\mathrm{E} 2$ threshold for $\mathrm{GnRH}$ sensitivity of the pituitary $\left(T_{E 2}^{G n R H, 2}\right)$. We also tested whether a decrease in the threshold of Inh for inhibition of FSH synthesis $\left(T_{I n h}^{F S H}\right)$, which results in a stronger inhibition of FSH synthesis by Inh, could result in a cycle with 2 waves. However, a decrease in $T_{I n h}^{F S H}$ did not change the pattern of 3 waves, and below 0.03 , no LH surges occurred. Although a large increase in $T_{I n h}^{F S H}$ resulted in a changed wave pattern, FSH and Inh levels became irregular such that we did not consider this as a normal 2-wave cycle.

In the model, the production of Inh was assumed to be proportional to Foll with a short delay, where $c_{\text {Foll }}^{I n h}$ is the proportionality factor of Foll to Inh. We assumed that FSH synthesis would be reduced when Inh production by the follicles was higher. Therefore, we tested whether an increase in $c_{\text {Foll }}^{\text {Inh }}$ would increase circulating Inh levels and result in a cycle with 2 waves, but this was not found.

P4 Synthesis. Another mechanism we tested was the inhibiting effect of $\mathrm{P} 4$ on follicle growth rate. We assumed that a 2-wave cycle could occur when a follicle wave can grow for longer due to less inhibition by $\mathrm{P} 4$. A reduced inhibitory effect on follicle growth rate could be due to a lower $\mathrm{P} 4$ production by the CL, resulting in

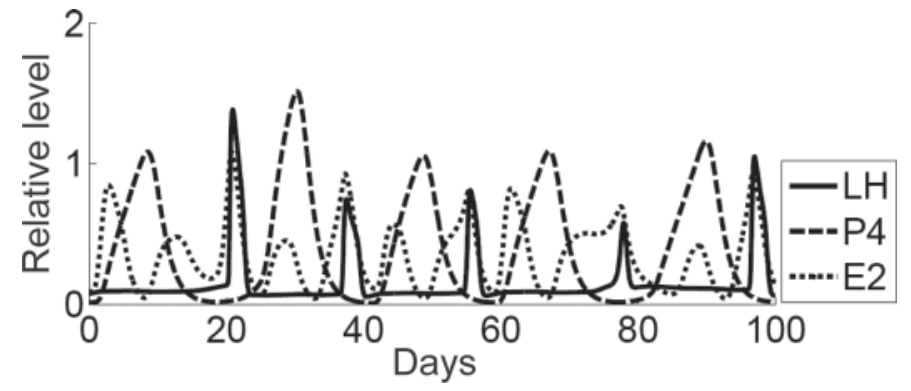

Figure 5. When the threshold of Foll to downscale FSH threshold $\left(T_{\text {Foll }}^{F S H}\right)$ was 0.12 , almost all cycles had 2 waves. $\mathrm{P} 4=$ progesterone, E2 $=$ estradiol.

lower P4 serum levels. In the model, it is assumed that P4 is proportional to CL function. Therefore, we tested whether a decrease of proportionality factor of CL to $\mathrm{P} 4\left(c_{C L}^{P 4}\right)$ would affect $\mathrm{P} 4$ levels and follicle growth rate. Simulation results did not show a clear effect of $\mathrm{P} 4$ levels on the time course of a follicular wave. A decrease in $c_{C L}^{P 4}$ resulted in lower peak $\mathrm{P} 4$ levels, but did not result in a 2-wave cycle. However, a decrease in $c_{C L}^{P 4}$ combined with a decrease in $m_{P 4}^{\text {Foll }}$ resulted in an interesting wave pattern. As described earlier, reduced inhibition of follicle growth rate by $\mathrm{P} 4$ (by reducing $m_{P 4}^{\text {Foll }}$ ) resulted in a 2-wave cycle when P4 serum levels were not changed. However, when P4 serum levels were reduced, by decreasing $c_{C L}^{P 4}$ from 1.45 to 0.50 , the wave pattern changed back to 3 waves (Figure 6). Intermediate values for $c_{C L}^{P 4}$ (between 0.50 and 1.45, in combination with $m_{P 4}^{F o l l}$ decreased to 1.00$)$ resulted in cycles with sometimes 3 , sometimes 2 waves. Thus, a parameter set was identified in which 2 parameter values were changed compared with the initial set that also simulates estrous cycles with 3 waves, but with different values for some of the output functions. In particular, E2 peak levels during nonovulatory waves in this new combination of parameter values for a 3 -wave cycle were higher than in the initial model parameterization for 3-wave cycles, possibly because of the reduced sensitivity of the follicles for P4. Further, the cycle length was a few days longer, probably because the lower P4 serum levels caused a later increase in OTR and thus CL regression. Therefore, a third wave could develop.

\section{Effects of Parameters Related to Time Point of CL Regression}

Besides the effect of follicle growth rate, we expected that the time point of CL regression would have an effect on the follicular wave pattern. Earlier CL regression could be due to an earlier increase of PGF $2 \alpha$. 
5994

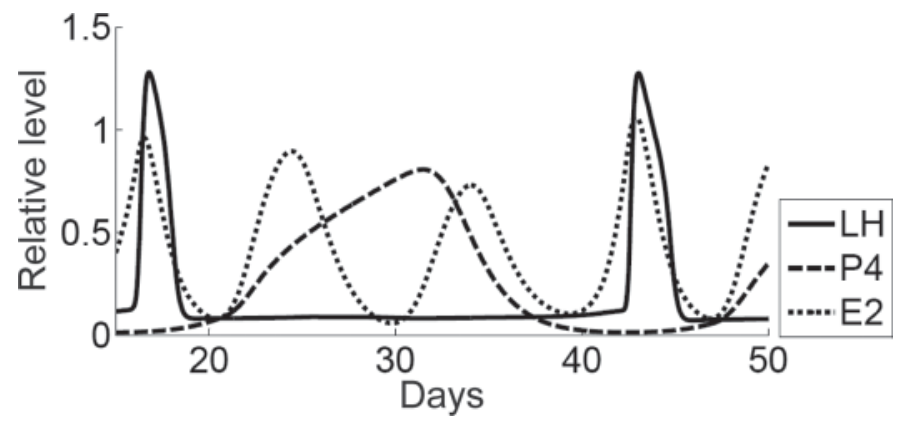

Figure 6. Three-wave cycle when the proportionality factor of CL to $\mathrm{P} 4\left(c_{C L}^{P 4}\right)=0.5$ and the maximum inhibition rate of $\mathrm{P} 4$ on Foll $\left(m_{P 4}^{\text {Foll }}\right)$ $=1.0 . \mathrm{P} 4=$ progesterone, $\mathrm{E} 2=$ estradiol.

Therefore, we tested whether a decreased threshold of OTR to stimulate $\mathrm{PGF}_{2 \alpha}$ increase $\left(T_{O T R}^{P G F \alpha}\right)$ could result in $\mathrm{PGF}_{2 \alpha}$ release at a lower OTR concentration and thus at an earlier time point, resulting in a cycle with 2 follicular waves. As expected, the simulation results showed that a change in time point of CL regression had a large effect on the number of follicular waves. A noteworthy result was that varying $T_{O T R}^{P G F 2 \alpha}$ could result in $1,2,3$, or 4 waves in a cycle (Table 3 ), because the time point of $\mathrm{PGF}_{2 \alpha}$ increase was changed. When the number of waves in a cycle increased, cycle length became longer and P4 levels higher, whereas E2 levels in the luteal phase became lower.

We tested whether a stronger P4 stimulation of OTR could also be obtained by an increased proportionality factor of P4 to OTR $\left(c_{P 4}^{O T R}\right)$. An increase in $c_{P 4}^{O T R}$ from 0.87 to 2.00 resulted in a cycle with 2 waves (Figure 7 ). The higher proportionality factor resulted in higher levels of OTR and lower levels of P4 compared with 3 -wave cycles. An overview of the differences in parameter values between 2 -wave and 3 -wave cycles is given in Table 2.

\section{Sensitivity Analysis}

The sensitivity analysis showed that the 3 parameters that easily induced a change in wave number $\left(T_{O T R}^{P G F 2 \alpha}, c_{P 4}^{O T R}\right.$, and $\left.m_{P 4}^{\text {Foll }}\right)$ were the most sensitive in the group of the 10 tested parameters. The parameters that did not induce a change in wave number had the lowest sensitivity in the group of tested parameters, whereas the parameters that needed an additional change in another parameter $\left(m_{F S H}^{F o l l}\right)$ or did not always result in a 2-wave cycle $\left(T_{F o l l}^{F S H}\right)$ had an intermediate sensitivity.

\section{General Discussion}

We used a mathematical model of the bovine estrous cycle to identify critical points in the mechanisms that

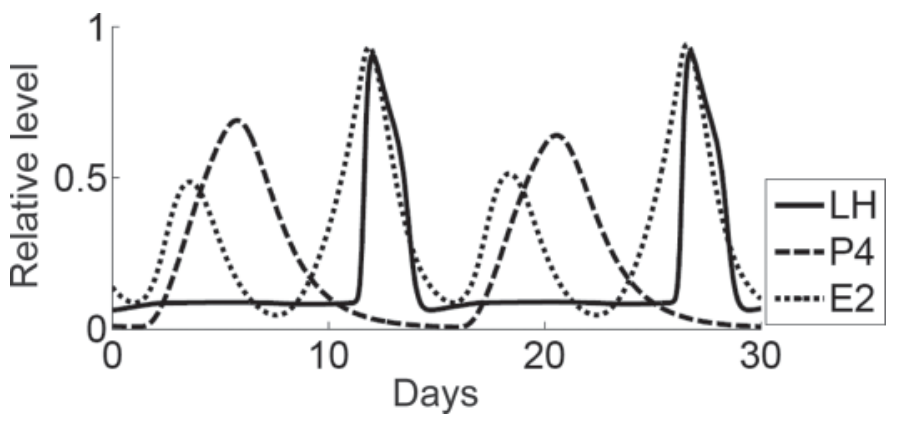

Figure 7. An increase in the proportionality factor of $\mathrm{P} 4$ to OTR $\left(c_{P 4}^{O T R}\right)$ resulted in a cycle with 2 waves. $\mathrm{P} 4=$ progesterone, $\mathrm{E} 2=$ estradiol.

affect the number of follicular waves in a cycle. We found that the model output changed from 3 to 2 waves in a cycle when the duration of the luteal phase was changed or when the effect of FSH or $\mathrm{P} 4$ on follicle growth was changed, but not when FSH synthesis or P4 synthesis was changed. Although some parameter changes resulted in irregular patterns, few simulations occurred in which the system was totally derailing or collapsing, and most of the times it recovered to a normal cycle, emphasizing the robustness of the model. Some parameterizations resulted in strictly periodic behavior of the cycle, whereas others resulted in quasiperiodic behavior. When a cycle had 2 waves instead of 3 , the duration of the cycle was shorter, which is in line with the empirical observations reported by Jaiswal et al. (2009), Ahmad et al. (1997), and Adams et al. (2008). The sensitivity analysis confirmed that parameters that affected the pattern of follicular waves indeed had a strong effect on the model solution.

Depending on the parameterization, the model simulations showed the same wave pattern repeatedly in successive cycles or alternating 2- and 3-wave cycles. In the literature, the repeatability of wave pattern within individual cows is studied in a limited number of papers. It was shown that wave pattern is repeatable within

Table 3. Overview of variations in the value of the threshold of oxytocin receptor (OTR) above which the stimulating effect on $\mathrm{PGF}_{2 \alpha}$ is increased $\left(T_{O T R}^{P G F 2 \alpha}\right)$ and the according number of waves per estrous cycle

\begin{tabular}{ll}
\hline Value of $T_{O T R}^{P G F 2 \alpha}$ & $\begin{array}{l}\text { Follicular } \\
\text { waves (no.) }\end{array}$ \\
\hline 0.25 & 1 \\
0.70 & 1 and 2 alternating \\
2.00 & 2 \\
2.50 & 2 and 3 alternating \\
4.10 & 3 \\
7.50 & 3 and 4 alternating \\
9.10 & 4 \\
\hline
\end{tabular}


individuals (Jaiswal et al., 2009) but also that cows can switch between cycles with 2 and 3 waves (Price and Carrière, 2004). Sichtar et al. (2010) reported an almost equal proportion of alternating and nonalternating patterns in cows monitored during 3 cycles. In a study of Rhodes et al. (1995) in 5 Bos indicus heifers monitored for at least 12 consecutive estrous cycles, 3 waves was the most common pattern. However, none of the cows showed 3 waves in all cycles; cycles with 2 and some with 4 waves were observed. Although the number of animals and consecutive cycles are limited, these studies suggest that both genetic and environmental factors may play a role in the regulation of follicular wave pattern.

For several single parameters, a shift in parameter value resulted in a cycle with 2 follicular waves instead of 3 . Although sometimes regular 2 -wave and 3 -wave cycles could be simulated for intermediate values, other values resulted in more irregular patterns, especially for parameters related to follicle growth rate. Possibly, slight changes in other parameters are required to "correct" for these irregularities, which indeed occurs in cows. This required change in other parameters could be demonstrated by the change in $m_{F S H}^{F o l l}$. An increase in $m_{F S H}^{\text {Foll }}$ combined with an increase in $T_{E 2}^{G n R H, 2}$, or a decrease in $m_{F S H}^{F o l l}$ combined with a decrease in $m_{P 4}^{F o l l}$ resulted in a 2-wave cycle.

A decrease in $c_{C L}^{P 4}$ alone did not change the number of waves, although $\mathrm{P} 4$ levels decreased. This could indicate that, for the regulation of the follicular wave pattern, the period in which $\mathrm{P} 4$ is produced is more important than the amount of $\mathrm{P} 4$ production (as long as $\mathrm{P} 4$ is above a minimum level). The simulation results clearly showed a gradual shift in the number of waves when $T_{O T R}^{P G F 2 \alpha}$ was changed. The time course of CL development and regression thus appears to have a distinct effect on the pattern of follicular waves, which is in line with the results of Jaiswal et al. (2009) who reported an earlier onset of CL regression in 2-wave cycles compared with 3 -wave cycles.

No regular 2-wave cycles could be simulated by changing parameters related to the effect of Inh on FSH synthesis rate. However, the simulation results showed that 2-wave cycles had higher Inh and FSH levels than 3 -wave cycles, which was also reported by Parker et al. (2003). However, doubling the amplitude of the FSH surge preceding the emergence of a follicular wave did not change the characteristics of that wave (Duggavathi et al., 2005). This suggests that FSH is normally not a limiting factor in follicle growth rate, but that changed Inh and FSH levels are a result rather than a cause of a changed follicular wave pattern. Although Foll in the model represents the capacity to produce E2 and Inh, it is correlated to follicle diameter. The higher levels of Foll in 2-wave cycles compared with 3 -wave cycles in the model output are therefore in line with the larger size of dominant follicles in 2-wave cycles compared with 3-wave cycles reported by Townson et al. (2002) and Wolfenson et al. (2004). The later emergence of the second wave in 2 -wave cycles compared with the initial 3 -wave cycle in the model is in line with the reports of Jaiswal et al. (2009) and Adams et al. (1992).

For parameters related to follicle growth rate, it was difficult to induce a smooth shift from 3 to 2 waves, because irregular patterns appeared in between. In contrast, for parameters related to the time point of CL regression, this gradual switch resulted less often in irregular patterns. These simulation results are in line with empirical observations in the literature reporting that the number of follicular waves can be manipulated easily by changing the time point of CL regression (Diskin et al., 2002). In this way, the third wave does not fit anymore within the amount of time. It is not surprising that an earlier time point of CL regression (and therefore a shorter cycle) induces a switch from 3 to 2 waves without irregular patterns in between, because when P4 levels are sufficiently decreased at the second wave, this will become the ovulatory wave. Although in the bovine, 2-wave cycles are on average shorter than 3-wave cycles (Bleach et al., 2004; Adams et al., 2008), the difference is not equivalent to the duration of a complete wave. Based on reported differences in follicle development (Bleach et al., 2004; Jaiswal et al., 2009), we postulate that differences in number of waves in natural estrous cycles may be due rather to changes in the mechanisms regulating follicle growth rate, and that the shorter cycle length is the result, not the cause, of the change in wave pattern. This is also shown by the simulation results because a change in follicle growth rate-related parameters, resulting in 2-wave cycles, led to a shorter cycle length.

\section{CONCLUSIONS}

The simulation results showed that several components of our model of the bovine estrous cycle could affect the pattern of follicle growth. The number of waves could be affected by follicle growth rate as well as time point of CL regression. The simulation results suggest that the initial number of waves per cycle is determined by certain characteristics of follicle growth rate, and indicates likely parameters that are involved in this mechanism. A better understanding of the endocrine mechanisms regulating follicle development is important to obtain more precise control of the estrous cycle, which could help to improve pregnancy rates (Diskin et al., 2002). Experimental data to verify the 
predicted causes of 2- or 3-wave cycles are not always available, but the present simulation results show some likely candidates involved in the regulation of follicle wave pattern that could be tested in future experiments. These experiments require daily measurements of follicle and CL dynamics and blood sampling for successive cycles. Additionally, P4 or FSH could be administered to measure specific effects. Designated experiments are extensive, but the present simulation results show some candidates to consider for deeper investigation. Therefore, our results allow experimental effort to be focused on these candidates.

\section{ACKNOWLEDGMENTS}

The authors thank P. Deuflhard (Department of Numerical Analysis and Modeling, Zuse Institute Berlin, Germany) for his contribution to the modeling work. These results are obtained through IP/OP: Systems Biology, which is financially supported by the Dutch Ministry of Agriculture, Nature and Food Quality (BAS no. 4434660700). C. Stötzel and S. Röblitz have been supported by the DFG Research Center Matheon "Mathematics for Key Technologies" in Berlin.

\section{REFERENCES}

Adams, G. P., R. Jaiswal, J. Singh, and P. Malhi. 2008. Progress in understanding ovarian follicular dynamics in cattle. Theriogenology 69:72-80

Adams, G. P., R. L. Matteri, J. P. Kastelic, J. C. H. Ko, and O. J. Ginther. 1992. Association between surges of follicle-stimulating hormone and the emergence of follicular waves in heifers. J. Reprod. Fertil. 94:177-188

Ahmad, N., E. C. Townsend, R. A. Dailey, and E. K. Inskeep. 1997. Relationships of hormonal patterns and fertility to occurrence of two or three waves of ovarian follicles, before and after breeding, in beef cows and heifers. Anim. Reprod. Sci. 49:13-28.

Bleach, E. C. L., R. G. Glencross, and P. G. Knight. 2004. Association between ovarian follicle development and pregnancy rates in dairy cows undergoing spontaneous oestrous cycles. Reproduction 127:621-629

Boer, H. M. T., C. Stötzel, S. Röblitz, P. Deuflhard, R. F. Veerkamp, and H. Woelders. 2011. A simple mathematical model of the bovine estrous cycle: Follicle development and endocrine interactions. J. Theor. Biol. 278:20-31.

Burns, D. S., F. Jimenez-Krassel, J. L. H. Ireland, P. G. Knight, and J. J. Ireland. 2005. Numbers of antral follicles during follicular waves in cattle: Evidence for high variation among animals, very high repeatability in individuals, and an inverse association with serum follicle-stimulating hormone concentrations. Biol. Reprod. $73: 54-62$
Deuflhard, P. 2004. Newton Methods for Nonlinear Problems: Affine Invariance and Adaptive Algorithms. In Springer Series in Computational Mathematics, Vol. 35. Springer-Verlag, Berlin, Germany.

Diskin, M. G., E. J. Austin, and J. F. Roche. 2002. Exogenous hormonal manipulation of ovarian activity in cattle. Domest. Anim. Endocrinol. 23:211-228.

dos Santos, R. M., M. D. Goissis, D. A. Fantini, C. M. Bertan, J. L. M. Vasconcelos, and M. Binelli. 2009. Elevated progesterone concentrations enhance prostaglandin F2 $\alpha$ synthesis in dairy cows. Anim. Reprod. Sci. 114:62-71.

Duggavathi, R., P. M. Bartlewski, E. Agg, S. Flint, D. M. W. Barrett, and N. C. Rawlings. 2005. The effect of the manipulation of follicle-stimulating hormone (FSH)-peak characteristics on follicular wave dynamics in sheep: Does an ovarian-independent endogenous rhythm in FSH secretion exist? Biol. Reprod. 72:1466-1474.

Fortune, J. E. 1994. Ovarian follicular growth and development in mammals. Biol. Reprod. 50:225-232.

Grah, A. 2004. Entwicklung und Anwendung modularer Software zur Simulation und Parameterschätzung in gaskatalytischen a Festbettreaktoren. PhD Thesis. Martin-Luther-Universität Halle-Wittenberg, Germany.

Jaiswal, R. S., J. Singh, L. Marshall, and G. P. Adams. 2009. Repeatability of 2-wave and 3-wave patterns of ovarian follicular development during the bovine estrous cycle. Theriogenology 72:81-90.

Ko, J. C. H., J. P. Kastelic, M. R. Del Campo, and O. J. Ginther. 1991. Effects of a dominant follicle on ovarian follicular dynamics during the oestrous cycle in heifers. J. Reprod. Fertil. 91:511-519.

Medan, M. S., T. Takedomi, Y. Aoyagi, M. Konishi, S. Yazawa, G. Watanabe, and K. Taya. 2006. The effect of active immunization against inhibin on gonadotropin secretions and follicular dynamics during the estrous cycle in cows. J. Reprod. Dev. 52:107-113.

Parker, K. I., D. M. Robertson, N. P. Groome, and K. L. Macmillan. 2003. Plasma concentrations of inhibin A and follicle-stimulating hormone differ between cows with two or three waves of ovarian follicular development in a single estrous cycle. Biol. Reprod. 68:822-828.

Price, C. A., and P. D. Carrière. 2004. Alternate two- and three-follicle wave interovulatory intervals in Holstein heifers monitored for two consecutive estrous cycles. Can. J. Anim. Sci. 84:145-147.

Rhodes, F. M., G. De'ath, and K. W. Entwistle. 1995. Animal and temporal effects on ovarian follicular dynamics in Brahman heifers. Anim. Reprod. Sci. 38:265-277.

Sichtar, J., R. Tolman, R. Rajmon, P. Klabanová, P. Berka, and J. Volek. 2010. A comparison of the follicular dynamics in heifers of the Czech Fleckvieh and Holstein breeds. Czech J. Anim. Sci. $55: 234-242$.

Silvia, W. J., G. S. Lewis, J. A. McCracken, W. W. Thatcher, and L. Wilson Jr. 1991. Review: Hormonal regulation of uterine secretion of prostaglandin F2 $\alpha$ during luteolysis in ruminants. Biol. Reprod. 45:655-663.

Townson, D. H., P. C. W. Tsang, W. R. Butler, M. Frajblat, L. C Griel Jr., C. J. Johnson, R. A. Milvae, G. M. Niksic, and J. L. Pate. 2002. Relationship of fertility to ovarian follicular waves before breeding in dairy cows. J. Anim. Sci. 80:1053-1058.

Wolfenson, D., G. Inbar, Z. Roth, M. Kaim, A. Bloch, and R. BrawTal. 2004. Follicular dynamics and concentrations of steroids and gonadotropins in lactating cows and nulliparous heifers. Theriogenology 62:1042-1055. 


\section{APPENDIX}

Table A1. List of model equations ${ }^{1}$

\begin{tabular}{|c|c|}
\hline $\begin{array}{l}\text { Equation } \\
\text { number }\end{array}$ & Equation \\
\hline 1. & $\frac{\mathrm{d}}{\mathrm{d} t} G n R H_{H y p o}(t)=\operatorname{Syn}_{G n R H}(t)-\operatorname{Rel}_{G n R H}(t)$ \\
\hline 1a. & $\operatorname{Syn}_{G n R H}(t)=c_{G n R H, 1} \cdot\left(1-\frac{G n R H_{H y p o}(t)}{G n R H_{H y p o}^{\max }}\right)$ \\
\hline $1 \mathrm{~b}$. & $\operatorname{Rel}_{G n R H}(t)=\left[H_{1}^{-}(P 4 \& E 2)+H_{2}^{-}(P 4)\right] \cdot G n R H_{\text {Hypo }}(t)$ \\
\hline 2. & $\frac{\mathrm{d}}{\mathrm{d} t} G n R H_{P i t}(t)=R e l_{G n R H}(t) \cdot H_{3}^{+}(E 2)-c_{G n R H, 2} \cdot G n R H_{P i t}(t)$ \\
\hline 3. & $\frac{\mathrm{d}}{\mathrm{d} t} F S H_{P i t}(t)=\operatorname{Syn}_{F S H}(t)-\operatorname{Rel}_{F S H}(t)$ \\
\hline 3a. & $\operatorname{Syn}_{F S H}(t)=H_{4}^{-}\left(\operatorname{Inh}_{\tau}\right)$ \\
\hline $3 \mathrm{~b}$. & $\operatorname{Rel}_{F S H}(t)=\left[H_{5}^{+}(P 4)+H_{6}^{-}(E 2)+H_{7}^{+}\left(G n R H_{P i t}\right)\right] \cdot F S H_{P i t}(t)$ \\
\hline 4. & $\frac{\mathrm{d}}{\mathrm{d} t} F S H_{\text {Blood }}(t)=\operatorname{Rel}_{F S H}(t)-c_{F S H} \cdot F S H_{\text {Blood }}(t)$ \\
\hline 5. & $\frac{\mathrm{d}}{\mathrm{d} t} L H_{P i t}(t)=\operatorname{Syn}_{L H}(t)-\operatorname{Rel}_{L H}(t)$ \\
\hline $5 \mathrm{a}$. & $\operatorname{Syn}_{L H}(t)=\left[H_{8}^{+}(E 2)+H_{9}^{-}(P 4)\right]$ \\
\hline $5 \mathrm{~b}$. & $\operatorname{Rel}_{L H}(t)=\left[b_{L H}+H_{10}^{+}\left(G n R H_{P i t}\right)\right] \cdot L H_{P i t}(t)$ \\
\hline 6. & $\frac{\mathrm{d}}{\mathrm{d} t} L H_{\text {Blood }}(t)=R e l_{L H}(t)-c_{L H} \cdot L H_{\text {Blood }}(t)$ \\
\hline 7. & $\frac{\mathrm{d}}{\mathrm{d} t} \operatorname{Foll}(t)=H_{11}^{+}(F S H)-\left[H_{12}^{+}(P 4)+H_{13}^{+}\left(L H_{B l o o d}\right)\right] \cdot \operatorname{Foll}(t)$ \\
\hline 8. & $\frac{\mathrm{d}}{\mathrm{d} t} P G F 2 \alpha(t)=H_{14}^{+}(O T R)-c_{P G F 2 \alpha} \cdot P G F 2 \alpha(t)$ \\
\hline 9. & $\frac{\mathrm{d}}{\mathrm{d} t} O T R(t)=c_{P 4}^{O T R} \cdot P 4(t)-c_{O T R} \cdot O T R(t)$ \\
\hline 10. & $\frac{\mathrm{d}}{\mathrm{d} t} C L(t)=H_{15}^{+}\left(L H_{\tau}\right)+H_{16}^{+}(C L)-H_{17}^{+}(P G F 2 \alpha) \cdot C L(t)$ \\
\hline 11. & $\frac{\mathrm{d}}{\mathrm{d} t} P 4(t)=c_{C L}^{P 4} \cdot C L(t)-c_{P 4} \cdot P 4(t)$ \\
\hline 12. & $\frac{\mathrm{d}}{\mathrm{d} t} E 2(t)=c_{F o l l}^{E 2} \cdot$ Foll $(t)-c_{E 2} \cdot E 2(t)$ \\
\hline 13. & $\frac{\mathrm{d}}{\mathrm{d} t} \operatorname{Inh}(t)=c_{\text {Foll }}^{\text {Inh }} \cdot$ Foll $(t)-c_{I n h} \cdot \operatorname{Inh}(t)$ \\
\hline
\end{tabular}

${ }^{1}$ For abbreviation of the notation of Hill functions, we used $H($ substrate $)$ instead of $m^{*} h[$ substrate $(t) ; T, n]$, where $n$ is the steepness coefficient. $H^{+}=$positive Hill function, $H^{-}=$negative Hill function, $T=$ threshold for change of behavior of the Hill functions, $m=$ parameter that controls the height of the switch of the Hill functions, Syn $=$ synthesis, Rel $=$ release, Pit $=$ pituitary, Hypo = hypothalamus, $c=$ rate constant, $\tau=$ time delay, Foll $=$ follicular function, $t=$ time, E2 $=$ estradiol, $P_{4}=$ progesterone, $I n h=$ inhibin, OTR $=$ oxytocin receptor, and $C L=$ corpus luteum. 
Table A2. List of Hill functions ${ }^{1}$

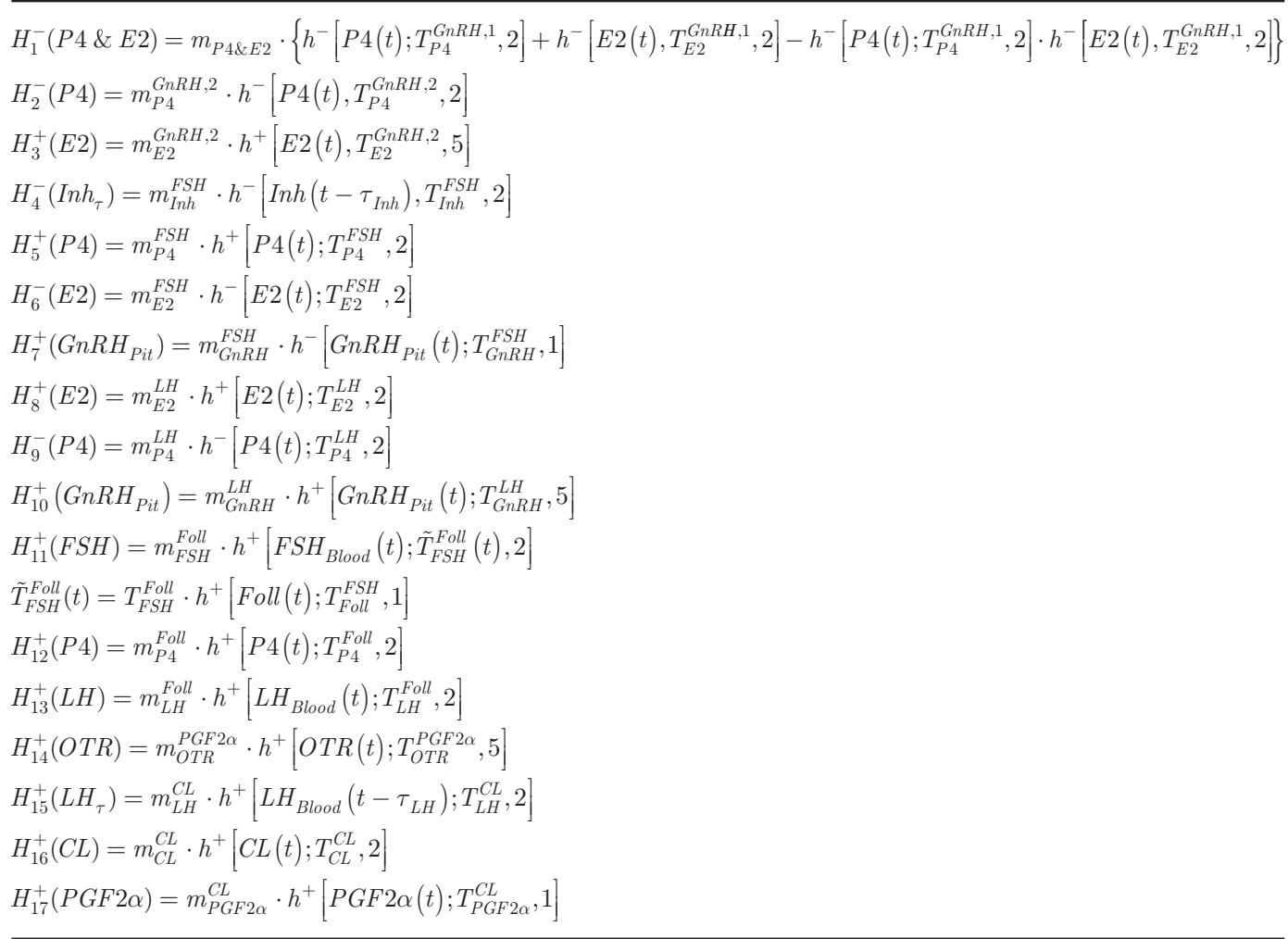

${ }^{1}$ See Tables 1 and A1 for abbreviations. 
Table A3. List of parameters and parameter values of the initial 3-wave cycle

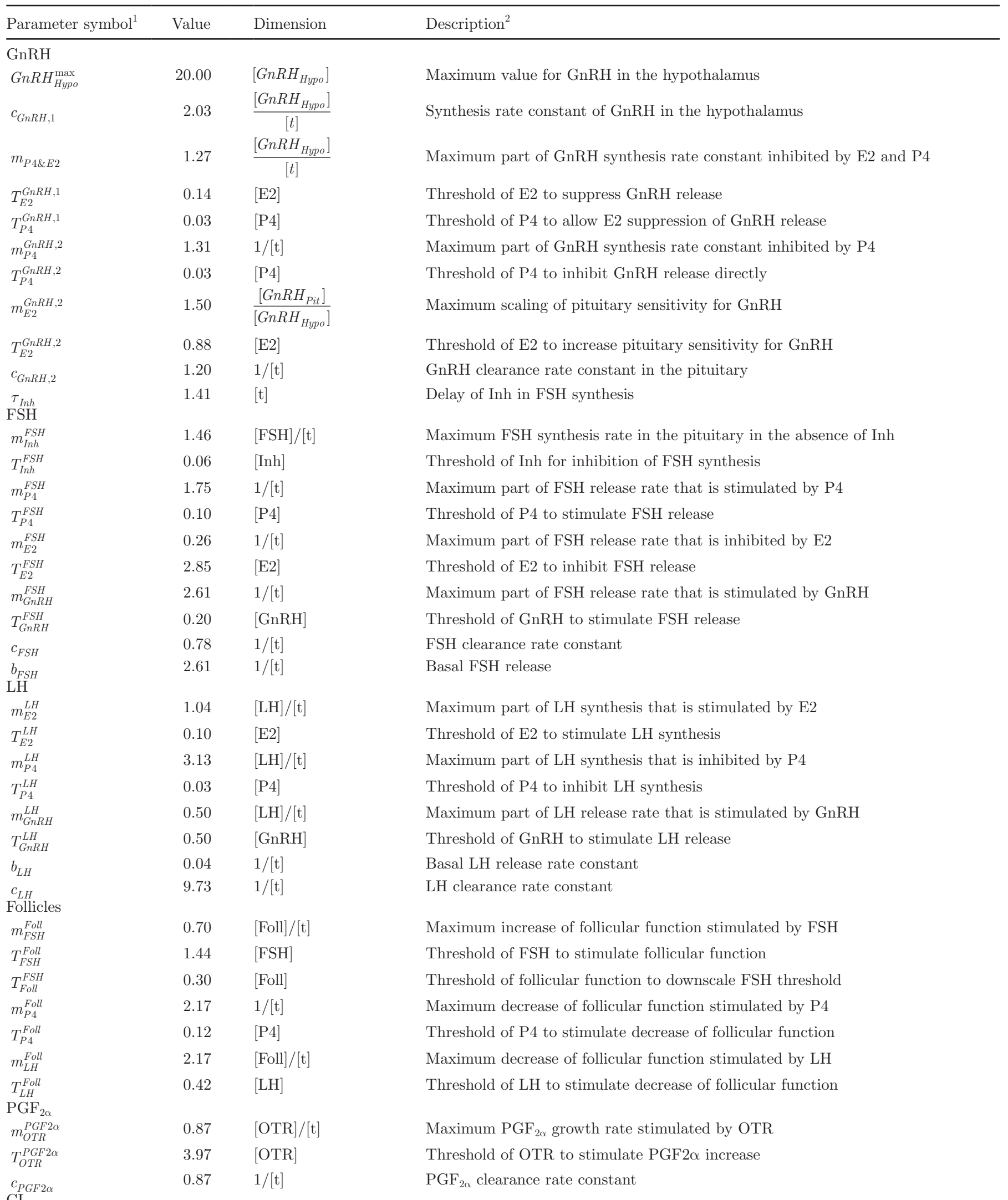


Table A3 (Continued). List of parameters and parameter values of the initial 3-wave cycle

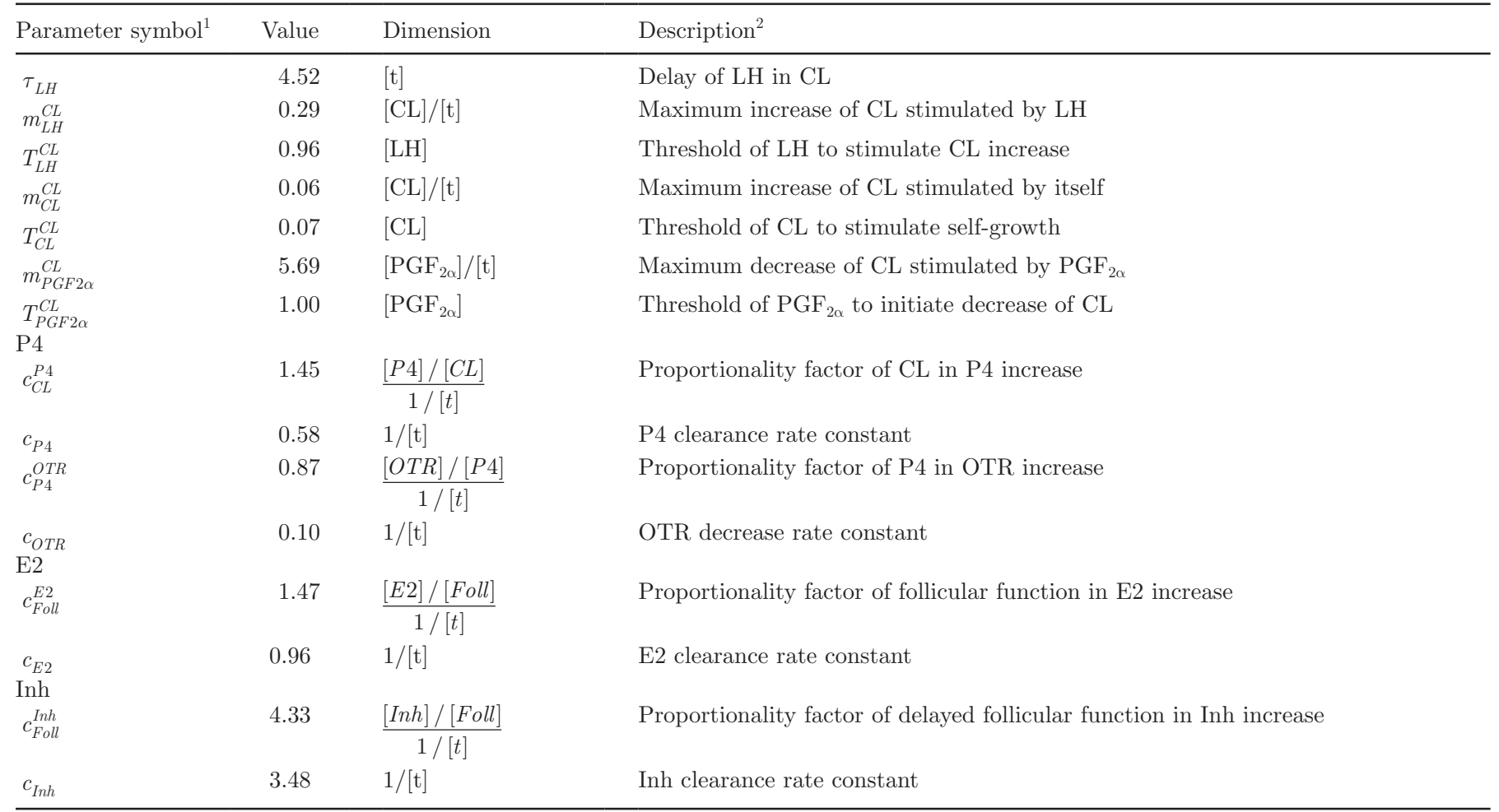

${ }^{1}$ See Tables 1 and A1 for definition of parameters.

${ }^{2} \mathrm{E} 2=$ estradiol, $\mathrm{P} 4=$ progesterone, $\mathrm{Inh}=$ inhibin, $\mathrm{OTR}=$ oxytocin receptor, $\mathrm{CL}=$ corpus luteum. 